\title{
The Energy Spectrum of Carriers between Two Concentric Spheres of Kane-Type Semiconductors
}

\author{
Arif Babayev, ${ }^{1,2}$ Sukru Cakmaktepe, ${ }^{2}$ and Deniz Turkoz ${ }^{2}$ \\ ${ }^{1}$ Institute of Physics, Azerbaijan Academy of Sciences, 370143 Baku, Azerbaijan Republic \\ ${ }^{2}$ Department of Physics, University of Suleyman Demirel, Isparta 32260, Turkey
}

Received 4 October 2005; Accepted 23 February 2006

Recommended for Publication by Michael Harris

\begin{abstract}
The electronic states of carriers between two concentric spheres of Kane-type semiconductor are theoretically investigated and compared with the results of the parabolic band approximation. Calculations are performed for a hard-wall confinement potential and the eigenstates and the eigenvalues of the Kane Hamiltonian are obtained. Taking into account the real band structure (strong spin-orbital interaction, narrow band gap), the size dependence of the energy of electrons, light holes, and spin-orbital splitting holes in InSb semiconductor concentric spheres are calculated. According to the obtained results both in parabolic and nonparabolic (Kane model) cases, the electron energy levels come close to each other with the increasing of the radius.
\end{abstract}

Copyright (C) 2006 Arif Babayev et al. This is an open access article distributed under the Creative Commons Attribution License, which permits unrestricted use, distribution, and reproduction in any medium, provided the original work is properly cited.

\section{INTRODUCTION}

In recent years, the optical properties of nanosize semiconductor crystals have attracted the attention of many investigators because of their potential applications $[1,2]$. The ability to tune their absorption and photoluminescence spectra over a very wide range of energy, as much as, $1.2 \mathrm{eV}$, by varying the crystal size opens the opportunity of fabricating nanocrystal-based tunable lasers and light-emitting diodes [3]. The progress in submicrometer technology has made possible the fabrication of new type of semiconductor nanostructures whose characteristics change with respect to the geometry of them as quantum dots, quantum wires, and quantum wells. Energy spectrum of carriers in quantum dots, quantum wires, and quantum boxes was investigated theoretically by many groups [3-6]. In [3], the size dependence of the electron and hole quantum size levels in spherical semiconductor nanocrystals were studied. In that work, an analytical theory of the quantum size levels within a spherical eight-band Pidgeon and Brown model, which takes into account both the coupling of conduction band and valence bands and the complex structure of the valence band in nanocrystals with an infinite potential barrier, was developed. The calculated level structures for narrow-gap InSb, moderate-gap CdTe, and wide-gap CdS nanocrystals were presented. It is shown that a new formalism for determining energy eigenstates of spherical quantum dots and cylindrical quantum wires in the multiple-band envelope-function approximation was described in [4]. The bound states were studied for the conduction band and coupled light and heavy holes, as a function of the radius of the GaAs/ $/ \mathrm{Al}_{x} \mathrm{Ga}_{1-x} \mathrm{As}$ quantum dot. Implications of the band-coupling effects for optical matrix elements for quantum wires and dots were discussed. The size dependence of the spectra of free carriers in $A^{3} B^{5}$ - and $A^{2} B^{6}$-type semiconductor spherical quantum dots is studied [5]. The advantages of a universal method for obtaining equations that are invariant under the transformations of the rotations groups, is that it directly yields a system of equations for the radial functions for any number of bands considered. A nonsymmetrized 8-band effective-mass Hamiltonian for quantum-dot heterostructures (QDHs) in Burt's envelope-function representation was derived [6]. The $8 \times 8$ radial Hamiltonian and the boundary conditions for the Schrödinger equation were obtained for spherical QDHs. Electron and hole energy spectra in three spherical QDHs, $\mathrm{HgS} / \mathrm{CdS}$; InAs/GaAs; and GaAs/AlAs, were calculated as a function of the quantum-dot radius within the approximate symmetrized and exact nonsymmetrized $8 \times 8$ models. In a study of Alonso et al., a relativistic free particle in a onedimensional box is studied [7].

In the present study, using three-band Kane's model including the conduction band, light, and spin-orbital splitting 
hole bands, the energy spectrum of carriers between two concentric spheres is calculated. The system considered here consists of two concentric spheres, and the carriers are assumed to be between the region of two concentric spheres. The potential of the concentric spheres is taken to be infinitive and consequently the wave functions are taken to be zero at the boundary.

In the three-band Kane's Hamiltonian, the valence and conduction bands interaction are taken into account via the only matrix element $P$ (so-called Kane's parameter). The system of Kane equations including the nondispersional heavy hole bands have the following forms $[8,9]$ :

$$
\begin{gathered}
-E C_{1}-\frac{P k_{+}}{\sqrt{2}} C_{3}+\sqrt{\frac{2}{3}} P k_{z} C_{4}+\frac{P k_{-}}{\sqrt{6}} C_{5}-\frac{P k_{z}}{\sqrt{3}} C_{7}-\frac{P k_{-}}{\sqrt{3}} C_{8}=0 \\
-E C_{2}-\frac{P k_{+}}{\sqrt{6}} C_{4}+\sqrt{\frac{2}{3}} P k_{z} C_{5}+\frac{P k_{-}}{\sqrt{2}} C_{6}-\frac{P k_{+}}{\sqrt{3}} C_{7}+\frac{P k_{z}}{\sqrt{3}} C_{8}=0 \\
-\frac{P k_{-}}{\sqrt{2}} C_{1}-\left(E+E_{g}\right) C_{3}=0, \\
\sqrt{\frac{2}{3}} P k_{z} C_{1}-\frac{P k_{-}}{\sqrt{6}} C_{2}-\left(E+E_{g}\right) C_{4}=0 \\
\frac{P k_{+}}{\sqrt{6}} C_{1}+\sqrt{\frac{2}{3}} P k_{z} C_{2}-\left(E+E_{g}\right) C_{5}=0 \\
\frac{P k_{+}}{\sqrt{2}} C_{2}-\left(E+E_{g}\right) C_{6}=0, \\
\frac{P k_{z}}{\sqrt{3}} C_{1}-\frac{P k_{-}}{\sqrt{3}} C_{2}-\left(\Delta+E+E_{g}\right) C_{7}=0 \\
-\frac{P k_{+}}{\sqrt{3}} \cdot C_{1}+\frac{P k_{z}}{\sqrt{3}} C_{2}-\left(\Delta+E+E_{g}\right) C_{8}=0
\end{gathered}
$$

Here $P$ is the Kane parameter, $E_{g}$ is the band gap energy, $\Delta$ is the value of spin-orbital splitting, and $k_{ \pm}=k_{x}+i k_{y}, \vec{k}=i \vec{\nabla}$, and $c_{i}$ are envelope functions.

\section{CALCULATING THE ENERGY SPECTRUM}

Substituting the expressions (3)-(8) into formulas (1) and (2), we obtain

$$
\left\{-E-\frac{p^{2}}{3}\left[\frac{2}{E+E_{g}}+\frac{1}{E+E_{g}+\Delta}\right] \Delta_{3}\right\} C_{1,2}=0,
$$

where $\Delta_{3}$ is three-dimensional Laplacian in spherical coordinates,

$$
\Delta_{3}=\frac{1}{r^{2}} \frac{\partial}{\partial r}\left(r^{2} \frac{\partial}{\partial r}\right)+\frac{1}{r^{2} \sin \theta} \frac{\partial}{\partial \theta}+\frac{1}{r^{2} \sin ^{2} \theta} \frac{\partial^{2}}{\partial \phi^{2}} .
$$

In spherical coordinates, the eigenfunction is

$$
C_{1,2}=R_{1,2}(r) Y_{l}^{m}(\theta, \phi),
$$

where $l$ is the orbital quantum number and $m$ is the azimuthal quantum number. The radial function $R_{1,2}(r)$ is found to satisfy the following differential equation:

$$
\begin{aligned}
& \left\{\frac{P^{2}}{3}\left(\frac{2}{E+E_{g}}+\frac{1}{E+E_{g}+\Delta}\right)\right. \\
& \left.\cdot\left(\frac{d^{2}}{d r^{2}}+\frac{2}{r} \frac{d}{d r}-\frac{l(l+1)}{r^{2}}\right)+E\right\} R_{1,2}(r)=0 .
\end{aligned}
$$

Equation (12) can be rewritten in the form

$$
\left(\frac{d^{2}}{d r^{2}}+\frac{1}{r} \frac{d}{d r}-\frac{l(l+1)}{r^{2}}+\chi^{2}\right) R_{1,2}(r)=0,
$$

where

$$
X^{2}=\frac{3}{P^{2}}\left(\frac{E\left(E+E_{g}\right)\left(E+E_{g}+\Delta\right)}{\left(3 E+3 E_{g}+2 \Delta\right)}\right) .
$$

The solution of (13) is spherical Bessel's equation and it is given by

$$
R_{1,2}(r)=A J_{l}\left(\mathcal{X}_{\mathrm{nl}} r\right)+B N_{l}\left(\mathcal{X}_{\mathrm{nl}} r\right)
$$

where $J_{l}\left(\mathcal{X}_{\mathrm{nl}} r\right), N_{l}\left(\mathcal{X}_{\mathrm{nl}} r\right)$ are the spherical Bessel functions of the first and second kinds. Two of the first spherical Bessel functions are as follows [10]:

$$
\begin{array}{cc}
J_{0}(r)=\frac{\sin r}{r}, & N_{0}(r)=-\frac{\cos r}{r}, \\
J_{1}(r)=\frac{\sin r}{r^{2}}-\frac{\cos r}{r}, & N_{1}(r)=-\frac{\cos r}{r^{2}}-\frac{\sin r}{r} .
\end{array}
$$

It is required that the wave function satisfy the following boundary condition:

$$
R_{1,2}\left(R_{1}\right)=R_{1,2}\left(R_{2}\right)=0 .
$$

Equation (17) yields the following result:

$$
J_{l}\left(X_{\mathrm{nl}} R_{1}\right) N_{l}\left(\mathcal{X}_{\mathrm{nl}} R_{2}\right)-J_{l}\left(\mathcal{X}_{\mathrm{nl}} R_{2}\right) N_{l}\left(\mathcal{X}_{\mathrm{nl}} R_{1}\right)=0 .
$$

Equations (14) and (17) together show that the radial eigenvalue spectrum is

$$
\left(\frac{E\left(E+E_{g}\right)\left(E+E_{g}+\Delta\right)}{\left(3 E+3 E_{g}+2 \Delta\right)}\right)=\left(\frac{z_{\mathrm{nl}}^{2}}{R^{2}}\right) \frac{P^{2}}{3},
$$

where $z_{\mathrm{nl}}$ is the $n$th root of (18).

Equation (19) determines the energies of electrons, light holes, and spin-orbital splitting holes in Kane-type semiconductor concentric spheres. It is also useful for analyzing the influence of nonparabolicity on the energy spectrum of carriers in concentric spheres.

In Figure 1, the dependence of $E(R)$ on two cases are presented: (a) electrons with parabolic dispersion law and (b) electrons with Kane's dispersion law for InSb concentric spheres. According to this figure, the energy levels in both cases become close to each other with the increasing of the inner radius $R_{1}$. At rather small sizes of $R_{1}$, the variance in the electron dispersion laws becomes more and more important, and therefore the curves for $E(R)$ keep away from each other. 


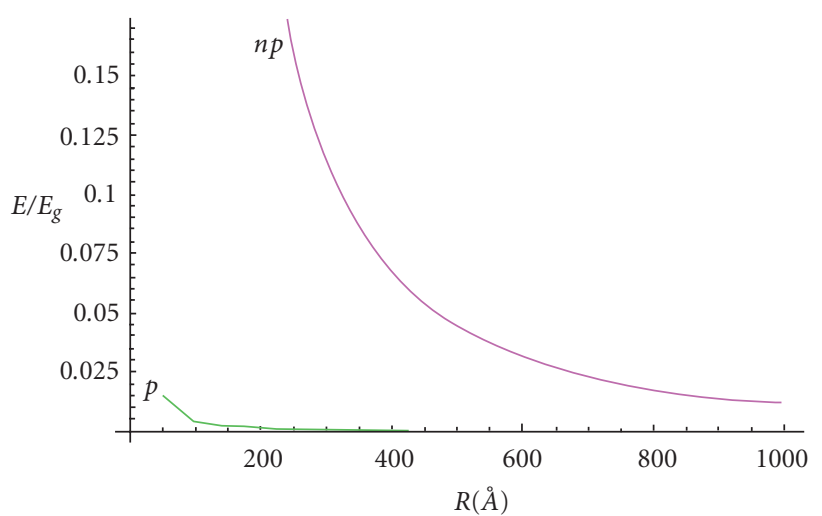

FIGURE 1: Lowest-order energy eigenvalues of electrons as a function of increasing inner radius $R_{1}$ for parapolic $(p)$ and nonparapolic $(n p)$ dispertion laws.

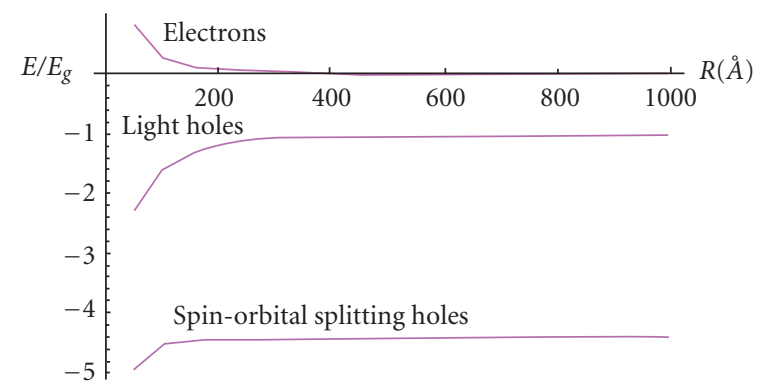

FIGURE 2: The energy spectrum of carriers (electrons, light holes, spin-orbital splitting holes) as a function of the inner radius $R_{1}$ in spherical quantum box for InSb.

In Figure 2, the inner radius $\left(R_{1}\right)$ dependence of the energy of carriers is given (electrons, light holes, spin-orbital splitting holes), where $R_{2} / R_{1}=2$ in nonparabolic dispersion law.

In Figure 3, the dependencies of $E(R)$ of electrons and light holes as a function of inner radius $R_{1}$, with $R_{2}$ fixed at 400 for nonparabolic dispersion laws, are presented for InSbtype concentric spheres. The energy scale is in units of $E_{g}, E_{g}$ is the band gap energy, and the curves are labeled by quantum numbers ( $\ln )$. According to this figure both in electrons and light holes for a small radius, there is an appreciable separation between states corresponding to different $n$ but equal $l$ quantum numbers.

In the calculations of the carriers energy spectra for narrow gap InSb concentric spheres, we chose the semiconductor band-structure parameters for InSb: energy gap $E_{g}=$ $0.2368 \mathrm{eV}$, spin-orbit splitting is $\Delta=0.810 \mathrm{eV}$, the value of the nonparabolicity parameter is $E_{P}=23.42 \mathrm{eV}$ [3].

\section{CONCLUSION}

The energy spectrum of carriers in InSb concentric spheres is investigated both in parabolic and nonparabolic dispersion laws. It is seen that the energy of electrons in parabolic and

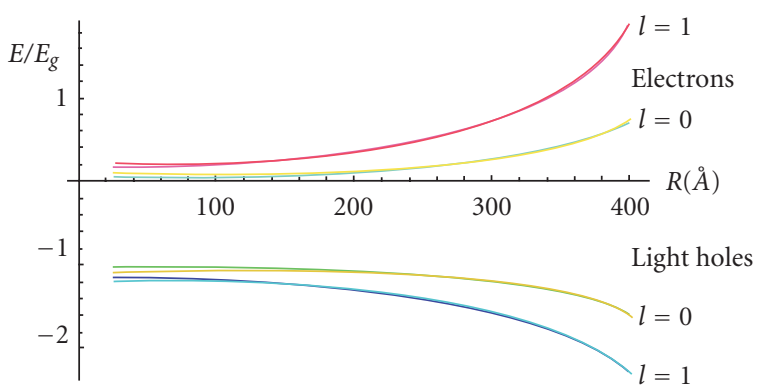

FIGURE 3: Lowest-order energy eigenvalues of electrons and light holes as a function increasing inner radius $R_{1}$ and outer radius $R_{2}=500$ for nonparabolic dispersion laws. The (ln) sequence is $\{(01),(0,2),(1,1),(1,2)\}$.

nonparabolic cases becomes close to each other with the increasing of the inner radius $R_{1}$. It is shown both in electrons and light holes that for a small radius, there is an appreciable separation between states corresponding to different $n$ but equal $l$ quantum numbers.

\section{REFERENCES}

[1] T. Chakraborty, Quantum Dots, Elsevier, Amsterdam, The Netherlands, 1999.

[2] D. Bimberg, M. Grundman, and M. Ledentsov, Quantum Dot Heterostructures, John Wiley \& Sons, New York, NY, USA, 2001.

[3] Al. L. Efros and M. Rosen, "Quantum size level structure of narrow-gap semiconductor nanocrystals: effect of band coupling," Physical Review B, vol. 58, no. 11, pp. 7120-7135, 1998.

[4] P. C. Sercel and K. J. Vahala, "Analytical formalism for determining quantum-wire and quantum-dot band structure in the multiband envelope-function approximation," Physical Review B, vol. 42, no. 6, pp. 3690-3710, 1990.

[5] F. M. Gashimzade, A. M. Babaev, and M. A. Bagirov, "Energy spectra of narrow- and zero-gap-semiconductor quantum dots," Journal of Physics: Condensed Matter, vol. 12, no. 36, pp. 7923-7932, 2000.

[6] E. P. Pokatilov, V. A. Fonoberov, V. M. Fomin, and J. T. Devreese, Journal of Physics: Condensed Matter, 1, vol. 12, no. 42, pp. 9037-9052, 2000.

[7] V. Alonso, S. De Vincenzo, and L. Mondino, "On the boundary conditions for the Dirac equation," European Journal of Physics, vol. 18, no. 5, pp. 315-320, 1997.

[8] T. Darnhofer and U. Rössler, "Effects of band structure and spin in quantum dots," Physical Review B, vol. 47, no. 23, pp. 16020-16023, 1993.

[9] A. I. Anselm, Introduction to Semiconductor Theory, Nauka, Moscow, Russia; Prentice-Hall, Englewood Cliffs, NJ, USA, 1978.

[10] R. L. Liboff, Introductory Quantum Mechanics, AddisonWesley, San Francisco, Calif, USA, 4th edition, 2002. 

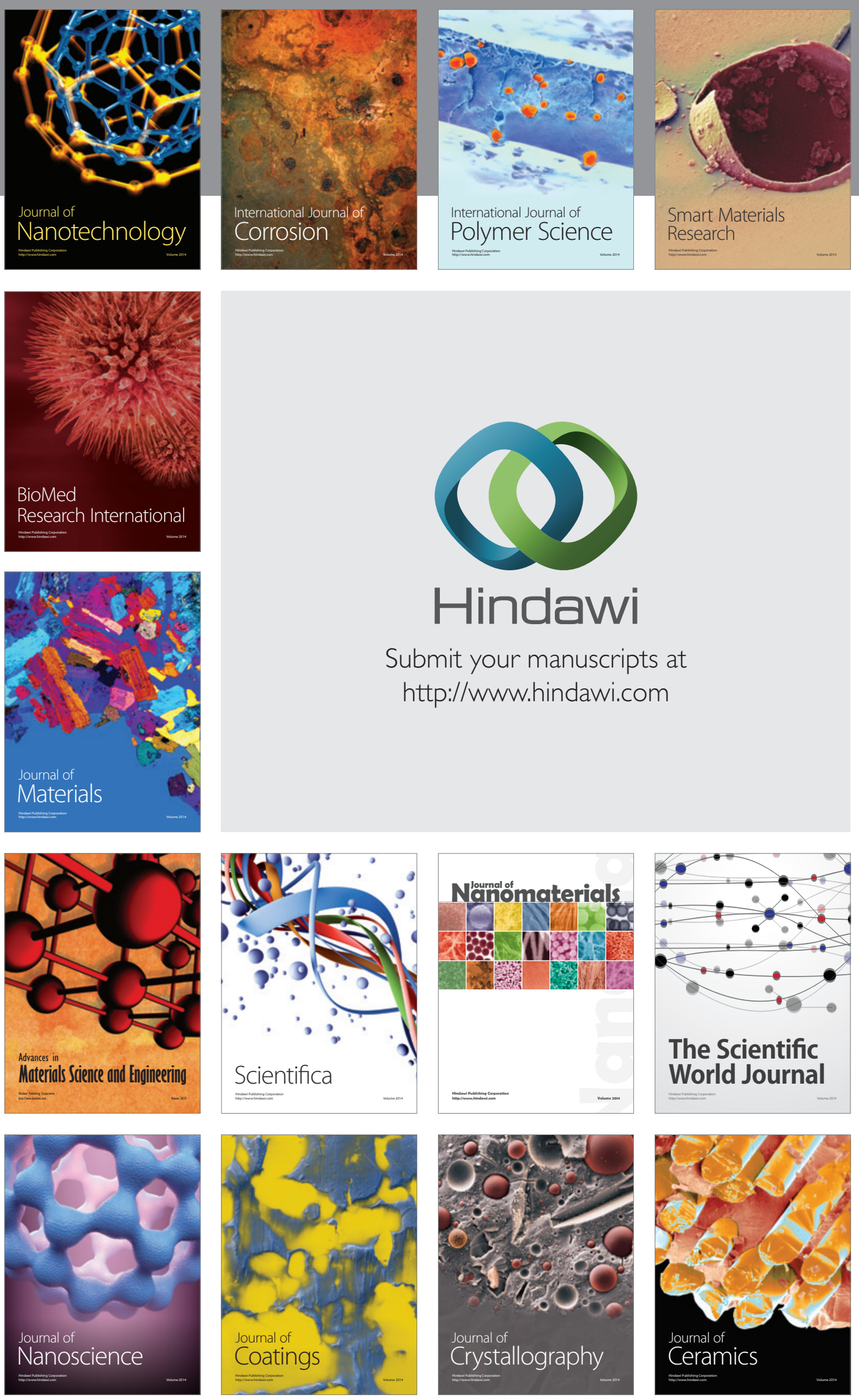

The Scientific World Journal

Submit your manuscripts at

http://www.hindawi.com

\section{World Journal}

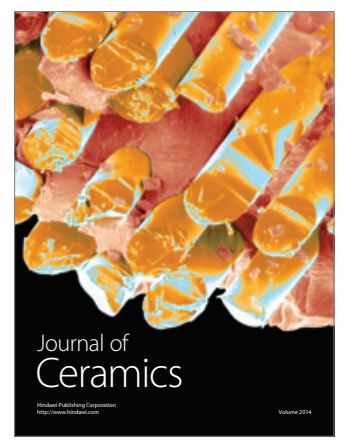

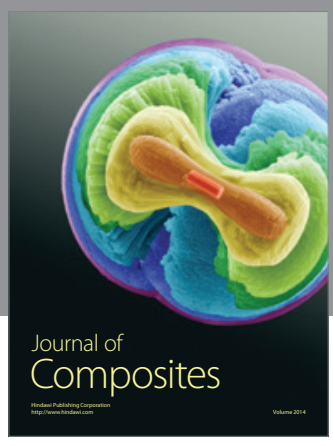
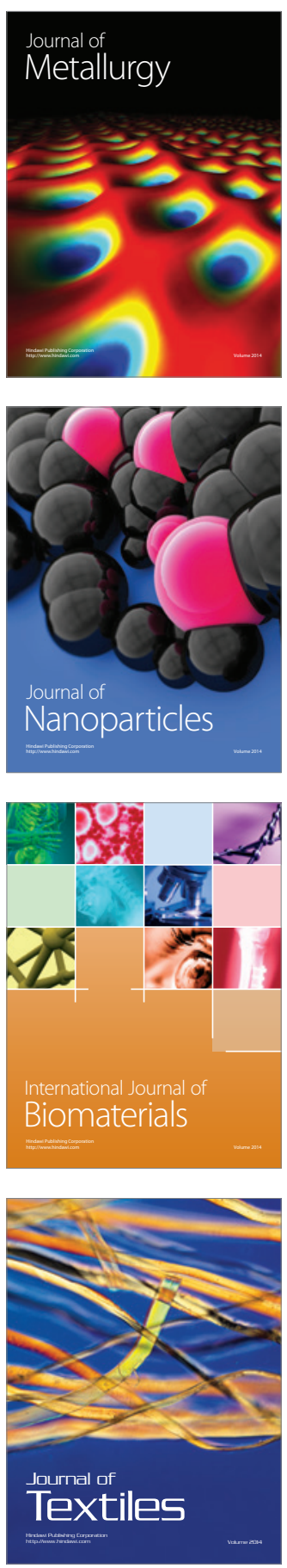